\title{
Ueber die Bestimmung des Eisenoxyduls in Silicaten.
} Von

\section{Dr. C. Doelter.}

Dass die Bestimmung, resp. Trennung der beiden Oxydationsstufen des Eisens für die Mineralógie von ganz besonderer Wichtigkeit ist, dürfte hinlänglich bekannt sein. Da die Mengen beider Oxyde oft in manchen Verbindungen ziemlich geringe sind und der Berechnung der Formeln wegen eine grosse Genauigkeit der Bestimmung nothwendig ist, so ist es unumgänglich, hier mehr als in irgend einem anderen Falle, Methoden anzuwenden, die grosser Genauigkeit zugänglich sind. Die Schwierigkeit exacter Trennung der beiden Oxydationsstufen des Eisens ist nun nirgends so gross als bei der Analyse der Silicate, dieser so complicirten and so wichtigen Gruppe des Mineralreiches, da hier noch die Schwierigkeit dazu kommt, die betreffende Substanz in Lösung zu bringen, ohne dass eine Reduction oder eine Oxydation vor sich geht.

Da die wenigsten Silicate in Schwefelsäure löslich sind, so muss gleichzeitig auf anderem Wege eine Aufschliessung bewerkstelligt werden. Hauptsächlich sind es 2 Methoden, die angewandt werden:

1) Díe Methode Hermann's durch Zusammenschmelzen mit Borax,

2) die Aufschliessung vermittelst Flusssäure und Schwefelsäure in zugeschmolzenen Glasröhren.

Was die erste Methode anbelangt, so ist sie wohl nicht ganz fehlerfrei; meistens tritt hierbei eine Reduction des Eisenoxyds ein, die oft nicht unbedeutende Abweichungen hervorbringt. Dies wurde namentlich von Suida*) durch eine grosse Anzahl von Versuchen gezeigt. Nach diesem gibt die Hermann'sche Methode stets zu grosse Mengen von Eisenoxydul.

Die besten Resultate liefert jedenfalls die zweite Methode: Aufschliessen mit Flusssäure in zugeschmolzenen Röhren. Die Verwendung von Salzsäure, die früher häufig stattfand, ist selbstverständlich beim Titriren mit Chamäleonlösung eine Quelle von Fehlern und muss vermieden werden. Man muss sich also der Schwefelsäure bedienen. Es zeigt aber die Erfahrung, dass die Resultate nur dann gut sind, wenn reine Flusssäure angewandt wird; nun ist aber sowohl die käufliche Flusssäure als auch die durch Herstellung vermittelst Flussspaths gewonnene

*) Mineral. Mittheilungen von Tschermak, Wien 1876; Heft III; und diese Zeitschrift 17, 212. 
stets unrein und muss zur Reinigung eine Destillation mit übermangansaurem Kali in einer Platinretorte vorgenommen werden; nur anf diese Weise dürfte ganz reine Flusssäure gewonnen werden können.

(E. L u d w ig.)

Bei dem Umstande, dass ich eine grosse Anzahl von Eisenoxydulbestimmungen in Silicaten auszuführen hatte, and eine Platinretorte zur Reinigung der Flusssäure mir nicht zu Gebote stand, labe ich eine andere Mothode angewandt, deren Princip mir von Prof. v. Pebal angegeben wurde, und welche ich hier kurz beschreiben möchte. *)

Vorerst aber gebe ich diejenigen Versuche, welche angestellt wurden, um die Fehler, die aus der Anwendung verunreinigter Flusssäure entstehen, zu ermitteln.

Es wurde vor Allem constatirt, dass die Flusssäure Chamäleonlösung entfärbte und zwar erforderten $2 c c$ käuflicher Flusssäure ungefähr 2-3 cc einer Chamäleonlösung von mässiger Concentration bis letztere nicht mehr entfärbt wurde.

Eine ähnliche Menge von krystall. Fluorammonium mit Schwefelsäure gemengt erforderte ebenfalls $2-3 c c$ derselben Chamäleonlösung. $2 c c$ einer aus Flussspath in einer Bleiretorte hergestellten Flusssäure erforderten circa 2,5 cc derselben Lösung bis zu dem Momente, wo keine Entfärbung des Chamäleons mehr eintrat, $2 c c$ vorker mit Chamäleon destillirter Flusssäure erforderten bei einem Versuche $0,4 c c$ der Lösung, bei anderen Versuchen trat keine Entfärbung der Lösung mehr 'ein.

Es handelte sich nun um fernere Versuche, um zu erkennen, ob durch einen früheren Zusatz von Chamäleon zu der Flusssäure nicht eine Rectificirung möglich wäre, jedoch ergibt sich aus folgenden Versuchen, dass dies nicht mit Genauigkeit ausführbar ist, sondern meistens eine Oxydation nicht verhindert wird; ferner aber, dass es sehr schwer ist, die richtige Menge Chamäleon von vornherein der Flusssäure zuzngiessen, damit die titrirte Lösung nicht mehr entfärbt werde; man kann leicht zu viel oder zu wenig Chamäleon zugiessen; gewöhnlich erhält man auf diese Weise etwas zu wenig Eisenoxydul, stets aber schwankende und unrichtige Resultate.

*) Aehnliche aber wohl nicht so exacte Methoden wurdén schon früher angewandt. (vergleiche Fresenius, quant. Analyse 6. Aufl. Bd. I. p. 576). 


\section{Versuchsreine.}

1) $0,239 \mathrm{~g}$ Eisendraht wurden auf die bekannte Weise mit Schwefelsäure im Kohlensäurestrom gelöst; diese Menge erforderte zur Titerbestimmung $45,5 \mathrm{cc}$ Chamäleon.

2) Dieselbe Menge wurde auf dieselbe Art gelöst, vorher jedoch circa $2 c c$ concentrirte, aus reinem Flussspath und Schwefelsäure bereitete Flusssäure hinzugesetzt; dieselbe Quantität Eisen erforderte nun $46,1 c c$ derselben Lösung.

3) Dieselbe Menge wurde auf gleiche Weise gelöst, wie oben bei (1), vorher jedoch der zugefügten Flusssäure etwas Chamäleon zugesetzt, bis keine weitere Fntfärbung stattfand; es waren in diesem Falle $44 \mathrm{cc}$ nothwendig.

4) Dieselbe Quantität wurde mit Flusssäure versetzt, der früher $0,4 g$ Chamäleon zugesetzt war, (so dass solche Flusssäure keine weitere Entfürbung des Chamäleons mehr hervorbrachte) und das Ganze wie bei der Methode der Eisenoxydulbestimmung in Silicaten in einem zugeschmolzenen Glasrohre behandelt. Zur Titrirung waren dieses Mal 44,6 cc erforderlich.

\section{Versuchsreihe.}

1) $0,189 \mathrm{~g}$ Eisendraht auf die bekannte Art gelöst erforderten . . . . . . . . . . . . . . . $68,2 c c^{*}$ )

2) Bei vorherigem Zusatze von käuflicher Flusssäure waren erforderlich . . . . . . . . . . . . . . $69,8 \ll$

3) Nachdem die Flusssäure mit $1 c c$ Chamäleon versetzt war, so dass letzteres keine Entfärbung mehr erlitt, waren nothwendig . . . . . . . . . . . . 66,5 (also im Ganzen $67,5 \mathrm{cc}$ ).

III. Versuchsreihe.

1) $0,0691 \mathrm{~g}$ Eisendraht auf obige Art gelöst erforderten $39,6 \mathrm{cc} * *$ )

2) Bei Zusatz von mit Chamäleon in einer Platinretorte destillirter Flusssäure waren erforderlich . . . . $39,2 \ll$ Wenn man Alles auf die zuletzt angewandte Lösung bezieht und die Mengen der angewandten Chamäleonlösung berechnet, so erhält man folgende Differenzen:

*) Daraus berechnet sich der Titer $1 c c=0,002771 \mathrm{~g}$ Eisen.

**) Fs ergibt sich für den Titer $1 c c=0,001745 \mathrm{~g}$ Eisen. 


$$
\begin{aligned}
& \text { für I. 2) . . . . . . . . }+1,8 \mathrm{cc} \\
& \ll \text { I. 3) . . . . . . . . }-4,5 \ll \\
& \ll \text { I. 4) . . . . . . . . }-2,7 \ll \\
& \text { « II. 2) . . . . . . . . }+2,6 \ll \\
& \text { « II. 3) . . . . . . . . }-2,8 \ll \\
& \text { «III. 2) . . . . . . . . }-0,4 \ll
\end{aligned}
$$

Man sieht also, dass bei Zusatz von Flasssäure stets zu viel Eisenoxydul sich ergibt; setzt man jedoch vor der Titration Chamäleon zu bis die Flusssäure nicht mehr entfärbt wird, so erhält man zu wenig Eisenoxydul. Daraus geht wohl hervor, dass jene früher unter 2) bezeichnete Methode nur dann zuverlässig ist, wenn die betreffende Flusssäure vorher mit übermangansaurem Kali destillirt wird, was den Besitz eines kostspieligen Platinapparates voraussetzt. Die von mir angewandte Methode vermeidet dies. Sie besteht wesentlich darin, nach Aufschliessung des Minerals vermittelst Flusssäure und Schwefelsäure, erstere zu verjagen. Auf diese Art fallen die durch die Flusssäure und ihre Verunreinigungen, schweflige Säure and Schwefelwasserstoff, sich ergebenden Fehlerquellen weg.

Der Apparat muss nun so construirt sein, dass weder eine Oxydation noch eine Reduction der vorhandenen Eisenverbindungen stattfinden kann. Die Aufschliessung geschieht in einem Platintiegel, in dem das feingepulverte Mineral nebst genügender Menge Flusssäure und Schwefelsäure behandelt wird. Die Operation geht in einer KohlensäureAtmosphäre vor sich; die Kohlensäure wird durch ein Glasrohr von oben in den Apparat geleitet, welcher unten luftdicht geschlossen ist. Der Apparat besteht aus einem flachen runden eisernen Teller, der an seinem Rande eine etwa $1 \frac{1}{2} \mathrm{~cm}$ vertiefte Rinne hat. Auf diesen wird der Platintiegel mit der Substanz gestellt.

Der Teller wird auf ein Wasserbad gesetzt, oder sonst von unten durch eine kleine Gasflamme erhitzt. Auf diesen Teller wird nun ein grosses hohes Becherglas*) mit dem offenen Theil gegen den Teller hingesetzt, so dass sein Rand in die oben erwähnte Rinne passt. Der Boden des Becherglases ist durchbohrt, so dass ein Rohr hindurchgehen kann. Die Kohlensäure wird von oben durch ein Glasrohr in den unteren Theil des Gefässes gebracht, so dass äie Röhre über dem Platintiegel endigt. Um einen Luftabschluss zu bewirken wird der Rand des

*) Die Verwendung eines solchen hat weiter keinen Nachtheil, als dass dasselbe ron Zeit zn Zeit zu ernenern ist. 
Becherglases entweder mit Sand oder einem anderen Verschlussmittel angefüllt; da eine starke Erhitzung nicht nothwendig ist, kann man sich auch des Quecksilbers bedienen, welches in die Rinne gegossen und wodurch ein vollkommener Luftabschluss erzielt wird. *)

Wenn es sich um Substanzen handelt, die leicht aufschliessbar siad, braucht man den Tiegel nur kurze Zeit zu erlitzen. Zwei Stunden genügen zur Aufschliessung und Verjagung der Flusssäure. Bei schwer aufschliessbaren Substanzen ist die Sache schwieriger, indem ein grösserer Tiegel nothwendig ist; soll Schwefelsäure nachgegossen werden, so kann man sich eines langen Trichterrohres bedienen. Anstatt eines Becherglases kann man auch einen Glascylinder verwenden, auf den oben eine gut anliegende durchbohrte Platte gelegt wird. Das Rohr, welches die Kohlensäure in den Apparat leitet, muss öfters erneuert werden.

Um die Methode zu erproben, wurden folgende Versuchsreihen gemacht :

I. Versuchsreihe.

Versuche mit Eisendraht.

1) $0,108 g$ feinen, reinen Eisendrahts wurden nach der gewöhnlichen Methode in einem Kolben mit Schwefelsäure im Kohlensüurestrom gelöst und mit Chamäleon titrirt, man gebrauchte . $75 \mathrm{cc}$

2) Ebenso wurden $0,0853 \mathrm{~g}$ Eisendraht behandelt und waren zur Titration erforderlich ${ }^{*}$ ) . . . . . . $59,4 \mathrm{cc}$

3) $0,0853 g$ Eisendraht wurden nach der von mir angegebenen Methode gelöst und titrirt, erforderlich . . 58,8 «

4) Nach letzterer Methode wurden behandelt $0,0605 \mathrm{~g}$, jedoch vorher Flusssäure zugesetzt und dieselbe in meinem Apparat verjagt, (Dauer des Experimentes circa 1 Stunde) es waren diesmal erforderlich . . . $42,8 \ll$

5) $0,0763 g$ Eisendraht wurden im Kölbchen gelöst und erforderten . . . . . . . . . . . . . . $53,5 *$ Daraus berechnet sich der Titer: $1 c c=0,001426 \mathrm{~g}$ Eisen.

6) $0,0862 g$ Eisendraht nach meiner Methode behandelt, in einer Schale unter der Glasglocke gelöst, erforderten $60,8 c c$ (Nach dem Titer berechnet wären erforderlich) . . 60,4 «

*) Nach oben hin braucht der Luftabschluss kein so vollkommener zu sein, da die Kohlensäure fortwährend eingeleitet wird and den ganzen unteren Theil des Apparates erfüllt; auf diese Art kann auch die Flusssäure aus dem oberen Theile des Gefässes entweichen.

${ }^{*}$ ) Aus diesen zwei Versuchen berechnet sich der Titer: 1 ce $=0,001436 \mathrm{~g}$ Eisen 
7) $0,0862 \mathrm{~g}$ Eisendraht wurden in einer Schale (wie bei 6) behandelt, vorher aber etwas Flusssäure zugesetzt und dieselbe verjagt; erforderlich waren diesmal . . $61,0 \mathrm{cc}$ theoretisch erforderlich . . . . . . . . . . 60,4

Daraus ergeben sich folgende Abweichungen von den berechneten Mengen (unter Zugrundelegung des Mittels von dem aus 1 und 2 berechneten Titer für die Versuche 3 und 4, und des aus 5 berechneten Titers für die beiden letzten Versuche):

$$
\begin{aligned}
& \text { für } 3 \text {. . . . . . }-0,6 c c \\
& \ll 4 \text {. . . . . . . }-0,6 \text { « } \\
& \ll 6 . .+. . \quad+0,4 \ll \\
& \text { * . . . . . . }+0,6 \ll
\end{aligned}
$$

II. Versuchsreibe.

Die Versuche wurden mit schwefelsaurem Eisenoxydul-Ammoniak ausgeführt.

1) $0,8343 g$ schwefelsaures Eisenoxydul-Ammoniak forderten bei der Titration . . . . . . . . . . . . . . . 119,5 co Daraus berechnet sich der Titer: $1 c c=0,0009997 \mathrm{~g}$ Eisen.

2) $0,3404 \mathrm{~g}$ in Wasser unter Zusatz von Schwefelsäure gelöst und längere Zeit in einer Schale in der Kohlensäure - Atmosphäre verdampft, erforderten bei der Titration . . . . . . . . . . . . . . . $49,4 c c$ berechnet nach dem Titer . . . . . . . . $48,8 \ll$

3) $0,705 \mathrm{~g}$ ebenso behandelt . . . . . . . . . $99,0<$ theoretisch notawendig . . . . . . . . . . 98,7

4) $0,431 \mathrm{~g}$ ebenso behandelt . . . . . . . . . $61,1 \ll$ theoretisch wären nothwendig . . . . . . . . 61,7 « Die Differenzen sind somit für 2 . . . . . $+0,6 c c$

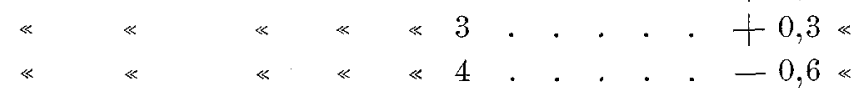

III. Versuchsreihe.

1) $0,8029 \mathrm{~g}$ schwefelsaures Eisenoxydul-Ammoniak direct tirirt . . . . . . . . . . . . . . . . $121,2 c c$ Titer : $1 c c=0,0009464 g$ Eisen. 
56 Doelter: Ueber die Bestimmung des Eisenoxyduls in Silicaten,

2) $0,235 \mathrm{~g}$ wurden mit Schwefelsäure und Wasser gelöst, die Lösung nach Zusatz von Flusssäure längere Zeit in der Kohlensäure-Atmosphäre verdampft, dann titrirt, erforderlich . . . . . . . . . . . . 36,200 theoretisch nothwendig wären . . . . . . . $35,5 \ll$

3) $0,435 \mathrm{~g}$ wurden auf die zuletzt angegebene Weise behandelt und erforderten . . . . . . . . . 64,8« theoretisch nothwendig wären . . . . . . . 65,4

Stellen wir die Differenzen zusammen, so ergeben sich:

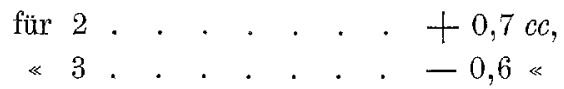

IV. Versuchsreihe.

Die folgenden Versuche wurden mit Fassait (grüner Augit von Fassa) angestellt und ergeben die Unterschiede zwischen der von mir gebrauchten Methode uad der der Aufschliessung mit Flusssäure und Schwefelsäure in zugeschmolzenen Glasröhren.

1) $0,54 \mathrm{~g}$ Fassait wurden in einer zugeschmolzenen Röhre mit Schwefelsäure und gewöhnlicher Flusssäure behandelt, sie erforderten von der Chamäleonlösung . . . . . . . . . . . . . $2,5 \mathrm{cc}$ entsprechend . . . . . . . . . . . . . . $1,52 \%$.

2) $0,532 \mathrm{~g}$ wurden ebenso behandelt, gebraucht . . . 2,7 cc entsprechend . . . . . . . . . . . $1,61 \%$.

3) $0,799 g$ wurden im Platintiegel in der KoblensäureAtmosphäre unter Zusatz von Schwefelsäure und Flusssäure aufgeschlossen, erforderlich waren von der betreffenden Lösung . . . . . . . . . . . . $5,8 \mathrm{cc}$ entsprechend . . . . . . . . . . . . . $1,87 \%$.

4) $0,821 g$ eines anderen Fassait-Materials in einer zugeschmolzenen Röhre mit Schwefelsäure und gewöhnlicher Flusssäure behandelt, ergaben . . . . . . . . $1,40 \ll$

5) $0,735 \mathrm{~g}$ von letzterem Material wurden ebenso behandelt, jedoch mit destillirter Flusssäure, es ergaben sich . . . . . . . . . . . . . $1,55 \ll$

6) $0,775 \mathrm{~g}$ desselben Materials wurden im Tiegel in der Kohlensäure-Atmosphäre unter Zusatz von gewöhnlicher Flusssäure behandelt und ergaben . . . . . . . 1,52 
Daraus folgt, dass beim Versuch 4, bei Anwendung von gewöhnlicher nicht gereinigter Flusssäure, eine bedeutende Abweichung sich kund gibt, während die zwei letzten Versuche gut übereinstimmen.

V. Versuchsreihe.

Durch die Versuchsreihe II und III wurde nachgewiesen, dass eine Oxydation des Eisenoxyduls, resp. des schwefelsauren Eisenoxyduls in dem von mir angewandten Apparate keineswegs stattfinden kamn; durch diese Versuchsreihen wurde auch nachgewiesen, dass ebensowenig eine Reduction eintritt. Um jedoch die Verhinderung letzterer im Apparate noch besser zu zeigen, habe ich anch Versuche mit Eisenoxyd, resp. mit schwefelsaurem Eisenoxyd und dessen Mischung mit schwefelsaurem Fisenoxydul angestellt.

1) $0,305 \mathrm{~g}$ Eisenoxydhydrat in Schwefelsäure gelöst ergaben keine Spur von Eisenoxydul; es trat nach längerem Verdampfen im Apparat keine Entfärbung des Chamäleons ein.

2) Eisenoxydhydrat in Schwefelsäure und. Flusssäure gelöst ergab nàch längerem Erhitzen im Apparat keinerlei Entfärbung der Chamäleonlösung.

3) $0,452 \mathrm{~g}$ geglühtes Eisenoxyd in Schwefelsäure gelöst erforderten nach der Lösung bei unmittelbarer Titration . . . $0,6 c c$ Nach längerem Verbleiben im Apparat ergab sich dieselbe Menge von Eisenoxydul.

4) Es wurde Eisenoxydhydrat in Schwefelsäure gelöst und dasselbe mit Eisenvitriol-Lösung gemengt; es wurden verbraucht bei der Titration einer solchen Lösung . 48 « (Titer: $1 c c=0,001436 \mathrm{y}$ Eisen.)

5) Von derselben Lösung wurde eine entsprechende Menge mit Schwefelsäure und Flusssäure in der KohlensäureAtmosphäre im Apparat erhitzt, und ergaben sich nach Berechnung der angewandten Menge auf die bei Versuch 4 angewandte . . . . . . . . . . . $48,6 \ll$

6) Letzterer Versuch mit einem andern Theil der bei den Versuchen (4) und (5) angewandten Lösung wiederholt, erforderte für dieselbe Menge . . . . . . 47,6 «

Aus dem bisher Bemerkten geht hervor, dass

1) bei Anwendung von nicht vorher mit Chamäleon destillirter Fluss- 
säure zur Aufschliessung von Silicaten, die erhaltene Menge von Eisenoxydul nicht ganz die richtige ist.

2) Dass durch Verjagung der Flusssäure bei der von mir beschriebenen Aufschliessungsmethode weder eine Oxydation noch eine Reduction eintritt, folglich die so bestimmte Menge von Eisenoxydul der wirklich vorhandenen entspricht, indem die $\mathrm{Ab}$ weichungen von letzterer als unbedeutende und nicht in einem bestimmten Sinne differirende sich ergeben; es erscheint demnach die Methode bei Anwendung der angedeuteten Vorsichtsmaassregeln für nicht allzu schwer aufschliessbare Silicate wie Hornblende, Augite etc. empfehlenswerth, und dürfte dieselbe für diejenigen Analytiker, welche keine Platinapparate zur Destillation der Flusssäure besitzen, empfehlenswerth sein, auch hat sie vor anderen sonst brauchbaren Methoden noch die einfache und rasche Anwendungsart voraus.

Graz, im Julj 1878.

\section{Analyse des Zsadányer Meteoriten. \\ Von}

\section{Dr. Wilhelm Pillitz.}

So zahlreich die bisher publicirten Meteorit-Analysen sind, ebenso selten begegnen wir einer detaillirten und systematisch geordneten Angabe des analytischen Ganges, der bei den einzelnen Analysen befolgt wurde. Zum grossen Theile finden wir Beschreibungen über die morphologische und mineralogische Natur, über die äussere Form des $\mathrm{Me}$ teoriten, ferner Umständliches über Ort und Zeit des Falles etc. In Bezug auf die Art und Weise, wie man zu den Resultaten der Analyse gelangt ist, müssen wir in der Regel mit einigen kurzen Bemerkungen vorlieb nehmen. Dieser Mangel an einem Wegweiser erschwert aber die Arbeit ungemein, indem man vorkommenden Falles gezwungen ist, durch langwierige und zeitraubende Voruntersuchungen erst die Wege ausfindig zu machen, die zum Ziele führen. Es dürfte daher nicht überflüssig sein, wenn ich im Folgenden den bei der Analyse des Zsadányer Meteoriten von mir eingehaltenen Gang ausführlich mittheile.

Eine nicht unbedeutende Schwierigkeit liegt zunächst in der genanem Trennung der gediegenen Metalle von den Metall-Oxyden und 